\title{
6 themes described patients' information needs related to patient controlled analgesia
}

Chumbley GM, Hall GM, Salmon P. Patient-controlled analgesia: what information does the patient want? J Adv Nurs 2002;39:459-71.

\section{QUESTION: What information do patients want about patient controlled analgesia (PCA)?}

\section{Design}

Focus groups. Findings were used to revise a patient information leaflet, which was subsequently evaluated in a randomised controlled trial (RCT). Only the findings of the focus groups are reported here.

\section{Setting}

\{St. George's Hospital NHS Trust, London, UK. $\}^{*}$

\section{Participants}

24 patients (19-72 y, 54\% women) were recruited from 8 surgical wards. All had used PCA during the previous week after major gynaecological, orthopaedic, abdominal, or renal surgery. Exclusion criteria were inability to ambulate, inability to speak or understand English, and unwellness.

\section{Methods}

7 focus groups were held. Patients were asked, "If you were going to use PCA for the first time, what would you want to know?" They were then asked to recommend changes to the content of a previously used information leaflet. Sessions were transcribed, and a thematic analysis was done. Topics were identified as themes when viewed as important by $>1$ focus group. The content of the new leaflet was based on themes from the first 5 focus groups $(n=15)$. Focus groups 6 and $7(n=9)$ reviewed first and second drafts of the new leaflet, respectively.

\section{Main findings}

6 themes were identified. (1) Patients worried about the dangers of morphine used in PCA, fearing addiction and overdose. Only 1 patient knew that PCA contained morphine, and the leaflet did not include this information. (2) Patients experienced various side effects of morphine, including fatigue, nightmares, and hallucinations, which sometimes caused them to stop PCA. The leaflet mentioned only nausea as a side effect. Patients felt it was important to identify other potential side effects so that they could seek help to alleviate them. (3) Patients were unaware that alternative pain relief to PCA was available if they were worried about morphine or experienced side effects. Some patients were confused when they were given alternative medication during weaning from morphine or to supplement PCA. (4) Patients felt confusion about how to use PCA and how it worked. When they awoke from the anaesthetic, some were simply told to "press the button" when they had pain. They didn't know how quickly morphine would work or how long each dose would last. (5) Patients felt a lack of support from staff. They felt they received contradictory instructions about the use of PCA and wanted detailed explanations from the pain team. (6) Patients talked about receiving information. Only 1 patient received the leaflet before surgery. Some patients would have liked an opportunity to discuss the leaflet with medical staff before surgery, and some would have preferred to have PCA explained to them. Others suggested that the pain team should bring a pump to the ward before surgery so that patients could become familiar with it. Some patients felt anxious because they didn't understand why the machine "bleeped". Other suggestions for the leaflet were that it include an illustration of the PCA machine or other diagrams, be printed in different languages, inform patients that PCA avoided the need for injections and that pain relief could be obtained without consulting nursing staff, and highlight important information in bold print.

\section{Conclusions}

Patients who used patient controlled analgesia (PCA) after surgery contributed to a new education leaflet after discussing their concerns and information needs about PCA, which included dangers of morphine, side effects, alternatives to PCA, confusion about how to use PCA, lack of support from staff, and receiving information.

*Information provided by author

\section{COMMENTARY}

The leaflet produced in the study by Chumbley et al differs from other leaflets on the same topic in the extent to which patients were involved in its design and evaluation to ensure that their expressed information needs were incorporated. Focus group methods, as used in this study, are gaining prominence as a way to better understand patient preferences. Furthermore, the combination of a qualitative approach with a quantitative evaluation strengthens the value of the findings.

A recent analysis contrasted the newer "patient empowerment" approach to creating printed patient information with the traditional "patient education" approach. ${ }^{1}$ The newer approach rejects a professional driven agenda of effecting change in patients' knowledge or behaviour in favour of enhancing choice in patients who are experts in their own needs and preferences. In the RCT by Chumbley et al, knowledge of PCA was enhanced in patients given the new leaflet. We must be sure, as in this case, that patients value such knowledge gains. Although patients found the new leaflet to be superior in terms of clarity and information about morphine, anxiety levels were not reduced. Staff should be aware of the value patients place on this information and be committed to provide not only the leaflets, but also the support and explanation that patients need. Future studies might address the effectiveness of leaflets supplemented by personal staff contact.

Processes must be developed to ensure that patient information is based on research findings, clinical expertise, and patient preferences. ${ }^{2}$ The study by Chumbley et al offers nurses insight into patient preferences related to PCA education, as well as a process for developing, implementing, and evaluating similar patient education leaflets.

Esther Coker, RN, MScN, MSc Clinical Nurse Specialist Hamilton Health Sciences Hamilton, Ontario, Canada

1 Dixon-Woods M. Writing wrongs? An analysis of published discourses about the use of patient information leaflets. Soc

2 Sci Med 2001;52:1417-32. based practice. Image J Nurs Sch 1999;31:317-22.
Source of funding: London Regional NHS Executive, Research and Development Directorate.

For correspondence: Dr G M Chumbley, Pain Management Centre, Charing Cross Hospital, London, UK gillian.chumbley@talk21.com 